\title{
Circulatory effects
}

It is almost trivial to state that the cardiovascular manifestations of shock are many and varied? Even in the attempts that have been made to find the locus of the primary change that migh $\vec{P}$ initiate all the others, attention has constantly shifted from one part of the cardiovascula $\overrightarrow{\mathrm{e}_{\mathrm{e}}}$ system to another.

For a long time the capillaries were held to be the site of the primary lesion; the heart ha $\frac{\Omega}{S}$ been implicated in some instances, as having vessels on the arterial and venous sides of the circulation between them. The original cause of the primary disturbance has been sought in toxins or in some aberration of the nervous control of the peripheral vasculature. Indeedoo the bewildered reader of all the accounts of the many investigations that have been carried out in this field might wonder if it will ever be possible to bring order to it. Dr Stalker's account of the microcirculation in shock shows a neat and simple way in which this can be done. Hiș lucid introduction justifies his definition of the process as essentially one of loss of micro带 circulatory control, with hypoperfusion. It is established that prolonged hypoxia leads to \& failure of contraction of the smooth muscle of arterioles and precapillary sphincters from whict they may never recover. The smooth muscle of venules is more resistant to a prolonged lacks of oxygen; hence capillaries dilate, flow is sluggish, and outward filtration of fluid predomin ates. Dr Stalker emphasizes the aggregation of red cells that may occur under these conditions and the obvious worsening of the situation which this phenomenon induces. This brings the rheological changes to the fore, and certain it is that they add a new dimension to the analysis. In particular the potential significance of fibrin microthrombi is being currentl emphasized, and platelet aggregation is a further phenomenon of real relevance. This last though not part of Dr Stalker's account, might come to dominate future investigations, as primary factor in haemorrhagic shock at least.

The paper which follows, by Dr Walters, emphasizes the low cardiac output of establishee shock, and the control of treatment designed to remedy this defect. He points to the inade quacies of attempts to measure blood volume and the difficulty of estimating what the norma blood volume of an individual patient should be. He recommends reliance on central venous pressure as an indication of the adequacy of the volume of circulating blood. It may be a pedan? tic point that the measurement actually required is that of central filling pressure rather that central venous pressure, but the latter is a very simple measurement to make and may be adequate for clinical purposes. It is clear, however, from Dr Walters' account that centras venous pressure is not sufficient on its own to assess the adequacy of a transfusion, thoug it does seem to enable a good measure of control to be achieved.

In his paper entitled 'Electronic and computer monitoring in shock' Dr Raison takes to the limits of what is now possible in the measurement of a wide variety of parameter廐 together with on-line computation of derived functions of possible importance. Quite apart from the cost effectiveness of the set-ups envisaged, or even the possibility of accommodating such large amounts of apparatus around a patient still allowing access to a nurse, one cann $\mathbb{F}^{\circ}$ help asking whether more time should not be spent investigating the useful application of these techniques as research tools before they are applied extensively on the ward.

Finally, Dr Flear takes us into rather different territory. He accepts that tissue hypoper fusion is the common feature of shock from a variety of causes, and then proceeds to exami the resulting changes that may occur at the cellular level, in particular the altered movements 
of sodium and potassium across the cell membrane, and their implications for shifts of water $\frac{\varrho}{\overline{5}}$ between the intracellular and extracellular compartments. His thesis is that these are the primary cellular events which are responsible for the changes in body water and other electrolytes which occur after trauma, and that they may have a valuable energy-sparing function.

The contributions in this section of the symposium thus cover a variety of topics relevant to the field, hypoperfusion of tissues perhaps providing the main idea common to them all. It only remains to ask whether hypoperfusion of the central nervous system might not add a further complication, quite apart from its possible significance for the recovery of adequate cerebral activity, by setting a limit on its own account to the recovery of cardiovascular function.

S. M. HILTON 